\section{Schmerzmedizin soll Pflichtfach im Studium werden}

Die Deutsche Gesellschaft für Schmerzmedizin fordert diese Disziplin als Prüfungsfach in die Approbationsordnung aufzunehmen. Außerdem müssten schmerzmedizinische Leistungen in den EBM integriert werden. Als ein Erfolg wertet die Fachgesellschaft, dass die Diagnose "Chronischer Schmerz" in den morbiditätsorientierten Risikostrukturausgleich aufgenommen worden ist. Das den Kassen für diese Versicherten zugewiesene Geld komme allerdings nicht bei den Ärzten an.

\section{Länder lassen sich Zeit mit effektivem Infektionsschutz}

Das 2011 in Kraft getretene Infektionsschutzgesetz, mit dem vor allem multiresistente Keime in Krankenhäusern und daraus resultierende schwere Nosokomialinfektionen bekämpft werden sollen, wird nur zögerlich umgesetzt. Diesen Vorwurf erheben die Deutsche Gesellschaft für Krankenhaushygiene e.V. (DGKH) und der FDP-Bundestagsabgeordnete Jens Ackermann. Die Länder sind verpflichtet, in eigenen Rechtsverordnungen die Details eines effektiven Infektionsschutzes zur regeln. Danach - so sei es zumindest in Brandenburg und möglicherweise auch in anderen Ländern geplant - sollen noch bis Ende 2016 Angestellte ohne Qualifikation als Hygienebeauftragte in Kliniken und Pflegeheimen eingesetzt werden können. Die DGKH verteidigt diese langen Fristen mit der Notwendigkeit, Hygienefachkräfte erst ausbilden zu müssen. Derzeit beschäftigen lediglich 5\% der Krankenhäuser über 400 Betten einen Hygienefacharzt.

\section{Spahn schlägt Egalisierung von GKV und PKV vor}

Der gesundheitspolitische Sprecher der CDU/CSU-Bundestagsfraktion, Jens Spahn, hat vorgeschlagen, die Trennlinie zwischen GKV und PKV aufzuheben und einen einheitlichen Versicherungsmarkt zu schaffen. Es sei nicht mehr zeitgemäß, dass sich nur Gutverdiener, Selbstständige und Beamte privat versichern können. Der stellvertretende Unionsfraktionsvorsitzende Johannes Singhammer wies dagegen auf den Koalitionsvertrag hin, wonach beide Zweige als Vollversicherung nebeneinander existieren sollen. Jedoch mussten auch Koalitionspolitiker jüngst eingestehen, dass die PKV wirtschaftliche Risiken in der Vollversicherung hat, vor allem durch säumige Mitglieder. Dies müsse die PKV aber selbst regeln, so Singhammer. $h l$

\title{
Frühe Nutzenbewertung: In fünf von sechs Fällen senkt der G-BA den Daumen
}

\author{
Auf der Basis der Empfehlungen des Instituts für Qualität und \\ Wirtschaftlichkeit im Gesundheitswesen (IQWiG) hat der Gemein- \\ same Bundesausschuss (G-BA) im März 2012 für sechs Wirkstoffe \\ über deren Zusatznutzen entschieden.
}

\section{Nur für Abirateronacetat $\left(Z^{2}\right.$ tiga ${ }^{\circledR}$ ) von} Janssen-Cilag (der Wirkstoff wird bei der Behandlung des metastasierenden Prostatakarzinoms eingesetzt) wurde für eine Patientengruppe ein Hinweis auf einen beträchtlichen Zusatznutzen gefunden.

2. Für Cabazitaxel (Jevtana ${ }^{\circledR}$ ) von Sanofi Aventis (zur Therapie vorbehandelter Patienten mit metastasierendem Prostatakarzinom bestimmt) entschied der G-BA, dass es für eine Patientengruppe einen Hinweis auf einen geringen $\mathrm{Zu}$ satznutzen gibt.

3. Der Zusatznutzen für den Wirkstoff Telaprevir (Incivo ${ }^{\circledast}$ ) von Janssen Cilag zur Behandlung von Hepatitis $\mathrm{C}$ ist gemäß G-BA nicht quantifizierbar.

4. Für das von Lilly und Boehringer Ingelheim angebotene Antidiabetikum Linagliptin (Trajenta ${ }^{\circledR}$ ) hält der G-BA einen Zusatznutzen wegen des nach seiner Ansicht unvollständigen Dossiers als nicht belegt. Hier gibt es zwischen Herstellern und G-BA Meinungsverschiedenheiten über die geeignete Vergleichstherapie. Der G-BA räumt den Unternehmen nun die Möglichkeit ein, ein Jahr nach Veröffentlichung des Beschlusses ein überarbeitetes Dossier vorzulegen und damit eine erneute Nutzenbewertung zu veranlassen.

5. Anhaltspunkte für einen geringen Zusatznutzen für Patienten mit rasch fortschreitender schwerer schubförmig-remittierender Multipler Sklerose sieht der G-BA bei Fingolimod (Gilenya ${ }^{\circledR}$ ) von Novartis. Aufgrund des erhöhten Risikoprofils und schwachen Nutzenbelegs hat der G-BA die Geltungsdauer seines Beschlusses auf drei Jahre befristet (siehe auch DNP 3/12).

6. Für den Wirkstoff Regadenoson (Rapiscan ${ }^{\circledast}$ ), ein Diagnostikum der Firma Rapidscan Pharma Solutions zur Darstellung von Herzfunktion und Herzdurchblutung, hat der G-BA aufgrund eines unvollständig einge- reichten Dossiers keinen Zusatznutzen als belegt angesehen. Auch hier kann das Unternehmen nach einem Jahr ein überarbeitetes Dossier einreichen, um das Verfahren erneut in Gang zu setzen.

\section{Hersteller und GKV-Spitzenver- band wollen Erstattungsbetrag}

Auf Basis der G-BA-Bewertungen treten nun Hersteller und der Spitzenverband der gesetzlichen Krankenversicherung in Verhandlungen über einen Erstattungsbetrag ein.

Bei Linagliptin - hier hatten die Hersteller einen Vergleich mit Sitagliptin bevorzugt, aber auch Daten zu der vom G-BA vorgegebenen Vergleichstherapie Metformin und Glibenclamid eingereicht - hat der G-BA aus formalen Gründen keinen Zusatznutzen gesehen. Das Angebot des G-BA, nach einem Jahr neu in die frühe Nutzenbewertung zu starten, hält der Deutschland-Chef von Boehringer Ingelheim, Dr. Engelbert Günster, für unzureichend. Er fordert, mit vorhandenen Daten und einem neu aufbereiteten Dossier unverzüglich in eine Neubewertung einzusteigen. Linagliptin wird wegen des ungewissen Ausgangs der Preisverhandlungen weiterhin nicht auf dem deutschen Markt angeboten.

Für Fingolimod, dem ersten oralen MSPräparat, hat der G-BA nur für eine Subgruppe von Patienten einen Hinweis auf einen geringen Zusatznutzen gesehen. Das Ergebnis dieser Bewertung sei „enttäuschend", so der Medizinische Direktor von Novartis, Professor Lothar Färber. Einen Teil der Daten aus Zulassungsstudien habe das IQWiG nicht bewertet, weitere, dem G-BA nachgereichte Informationen seien als unzureichend eingestuft worden. Aufgrund dieser Erfahrungen fordert Novartis einen regelmäßigen Austausch aller am Prozess Beteiligten.

Helmut Laschet 